\title{
Correction to: Land surface characterization using BeiDou signal-to-noise ratio observations
}

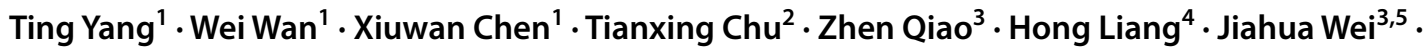 \\ Guangqian Wang ${ }^{3,5} \cdot$ Yang Hong ${ }^{1,5,6}$
}

Published online: 14 October 2019

○) Springer-Verlag GmbH Germany, part of Springer Nature 2019

\section{Correction to: GPS Solutions (2019) 23:32 https://doi.org/10.1007/s10291-019-0824-4}

In the original article publication, the affiliation of co-author Yang Hong was accidentally omitted from the manuscript. The missing affiliation is given below:

School of Civil Engineering and Environmental Science, University of Oklahoma, Norman, OK 73019, USA

Accordingly, the biography of Yang Hong is corrected as follows:
The original article can be found online at https://doi.org/10.1007/ s10291-019-0824-4.

\section{Wei Wan}

w.wan@pku.edu.cn

1 Institute of Remote Sensing and GIS, Peking University, Beijing, China

2 Conrad Blucher Institute for Surveying and Science, Texas A\&M University-Corpus Christi, Corpus Christi, TX, USA

3 State Key Laboratory of Plateau Ecology and Agricultural, Qinghai University, Xining, China

4 Center of Meteorological Observation, China Meteorological Administration, Beijing, China

5 Department of Hydraulic Engineering, Tsinghua University, Beijing, China

6 School of Civil Engineering and Environmental Science, University of Oklahoma, Norman, OK 73019, USA
Yang Hong received his B.S. and M.S. degrees from Peking University in 1996 and 1999, respectively, and received a Ph.D. degree from the University of Arizona in 2003. His research interests include radar/satellite remote sensing of the water cycle, hydrology and water resource, hydrometeorology, and hydroclimatology. 